\title{
Design, Development and Implementation of an Iot Based Intelligent Ambient Controller for Lvdc Enabled Green Buildings
}

\author{
Drisya K, Asha T S
}

\begin{abstract}
Energy efficiency of green buildings are improving its performance using Internet-of Things (IoT) based automation and associated developments. Also, as solar based power generation is achieving a decentralized structure, LVDC-alone power distribution improves efficiency and quality of power. Combining Green buildings, LVDC and IoT seems to be the future in energy management. An LVDC and IoT managed intelligent ambient light controller is developed to monitor human occupancy and thus reduce loss. LVDC based $12 v$ power rail is considered and are connected through publish-subscribe based MQTT IoT communication protocol. The master node measures the Ambient light and communicated to other nodes and which independently alters the attached PWM driven LEDs. Human presence is sensed using a microwave radar sensor and each node communicates its presence in MQTT. An algorithm is developed to control lighting based on ambient light and human presence.
\end{abstract}

Index Terms-Green building, IoT, LVDC, MQTT, PWM

\section{INTRODUCTION}

Green Building is the term that is used to indicate structures and processes that are environmentally responsible and efficient throughout the buildings life cycle. Green buildings are also known as sustainable or high performance buildings. Such buildings reduces the usage of water, optimizes the energy efficiency, conserves natural resources and generates less waste. Green buildings also provides healthier spaces for persons. Low Voltage Direct Current [LVDC] is the electricity for the $21^{\text {st }}$ century. LVDC technology accelerates energy access and improves energy efficiency. The various aspects of LVDC provides a facility to access the electricity even in the remote villages. The power can be generated in the place where it is consumed. The houses and buildings lightened by the public power distribution network are now able to generate power via solar panels and small wind turbines. With DC power generation, storage and consumption, each building is able to run autonomously. The advantages of $\mathrm{DC}$ over $\mathrm{AC}$ includes a number of points. The renewable energy resources produce current in DC. A large majority of electrical and electronic devices are using DC current. The DC energy generation requires low material cost, higher energy efficiency and reduced conversion losses.

The exact idea of this work is to realize a smart home system or a smart building system. A smart home system

Revised Manuscript Received on April 12, 2019.

Drisya K, PG Student, Department of Electronics and Communication Engineering, NSS College of Engineering, Palakkad, Kerala, India. (drisyak1995@gmail.com)

Asha T S, Professor, Department of Electronics and Communication Engineering, NSS College of Engineering, Palakkad, Kerala, India (ashapkumar@rediffmail.com) will control lighting, climate, entertainment systems and appliances. Home security such as access control and alarm systems are also included in smart home concept. Internet of Things [IoT] is the extension of internet connectivity into physical devices and everyday objects. The IoT has become an unavoidable component in recent works. In recent years, the major significant trend in IoT is the drastic growth in number of devices that can be connected. The IoT create opportunities for more direct integration of the physical world in to computer based systems, which leads to improvement in efficiency, economic benefits and reduction in human efforts.

\section{IOT BASED INTELLIGENT AMBIENT CONTROLLER}

Energy usage in the world is increasing day by day due to increasing demand of devices and new needs. The energy demand of the world is increase about $30 \%$ in 2035 . India's energy consumption is also set to grow $4.2 \%$ a year by 2035 . This growth is faster than that of all major economies in the world. With increase in energy consumption, the energy wastage is also increases. Continuous lighting of lamps even in the absence of persons in a room and in day time make large amount of energy to loss. So an IoT based intelligent ambient controller haS much importance in now-a-days.

Ambient controller refers to the electronic environments that are sensitive and responsive to the presence of people. Ambient controller enhance the way people interact with their environment and provides safety. The basic idea is that by enriching the environment with technology together with sensors and devices interconnected through a network, such a system can be useful for real time environment. When intelligence is added to the ambient controller, it becomes an Intelligent Ambient Controller.

In the designed system, three light modules are considered. The system is suited for a corridor or a large room. One light module is placed in the entrance of the corridor and other two modules are placed within the corridor far apart. The light modules should be placed atleast $1.5 \mathrm{~m}$ apart. The module placed at the entrance consist of a light sensor and a presence detector. The light sensor is the TSL2561 luminosity sensor and the presence detector used is the RCWL0516. Figure 1 shows the block diagram of the module 1 [one placed in the entrance] and the figure 2 represents the block diagram of module 2 and 3 [where light sensor is absent].

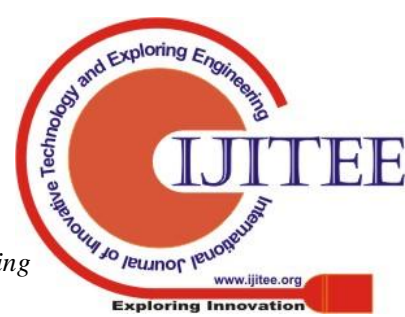




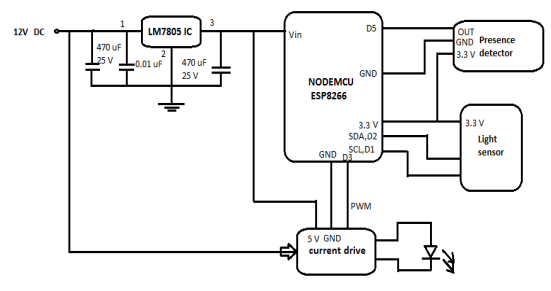

Fig 1. Block diagram of module 1

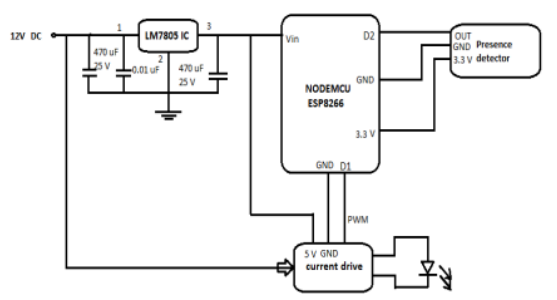

Fig 2. Block diagram of module 2 and 3

The block diagram consist of a microcontroller, current drive, voltage regulator IC, presence detector and a light sensor. The light sensor is optimal and present in the first module and is absent in remaining two. The light sensor in the module 1 senses the ambient light in lux and the information is passed to all the three modules via MQTT protocol. The light intensity is adjusted according to the measured lux value. Each module is embedded with a NodeMCU. It is one of the single board microcontroller developed by ESP8266 Open source community. The ESP8266 is capable of either hosting an application or offloading all $\mathrm{WiFi}$ networking functions from another application processor.

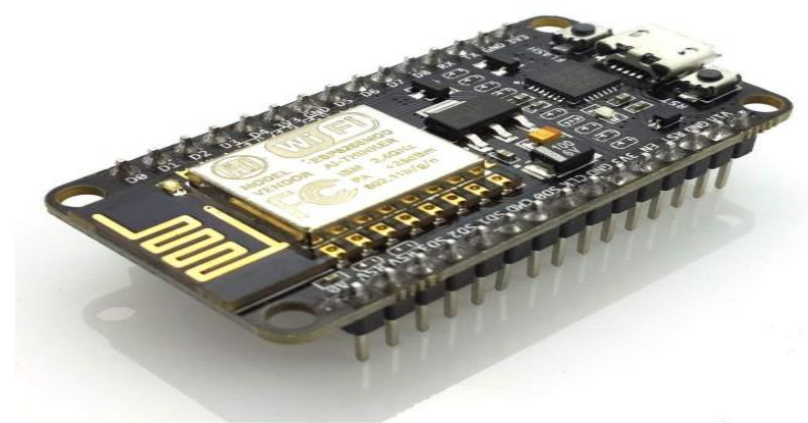

Fig 3.NodeMCUESP8266 module

The PWM pin of the NodeMCU is given to the current driver circuit. The circuit represents an excellent way of making a linear circuit regulator circuit using transistors.

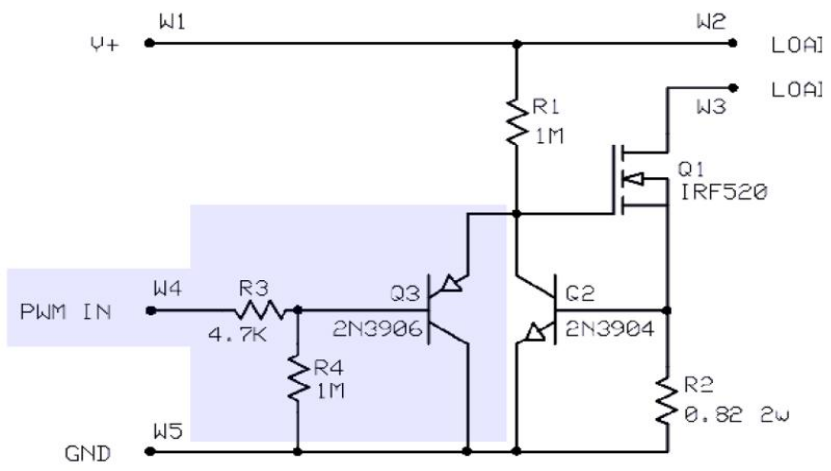

Fig 4. Current drive circuit

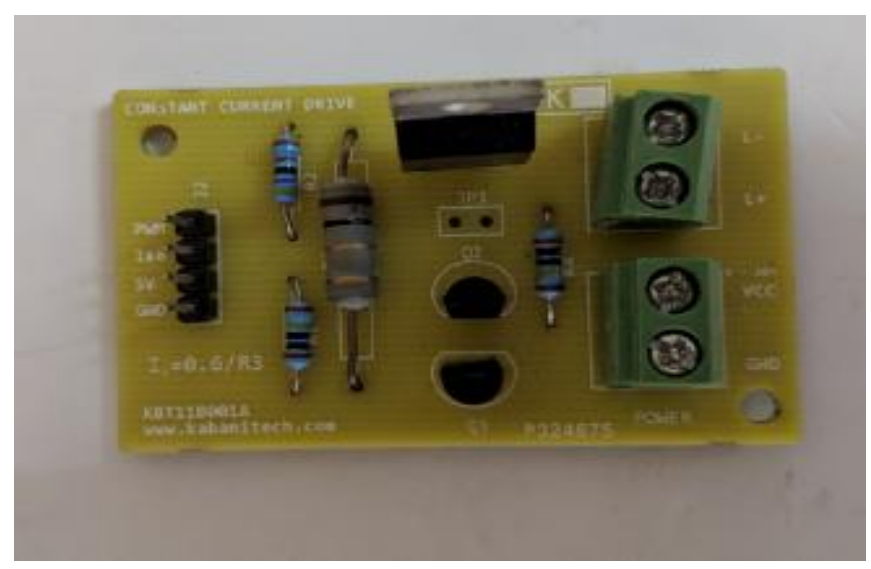

Fig 5. Current driver circuit board

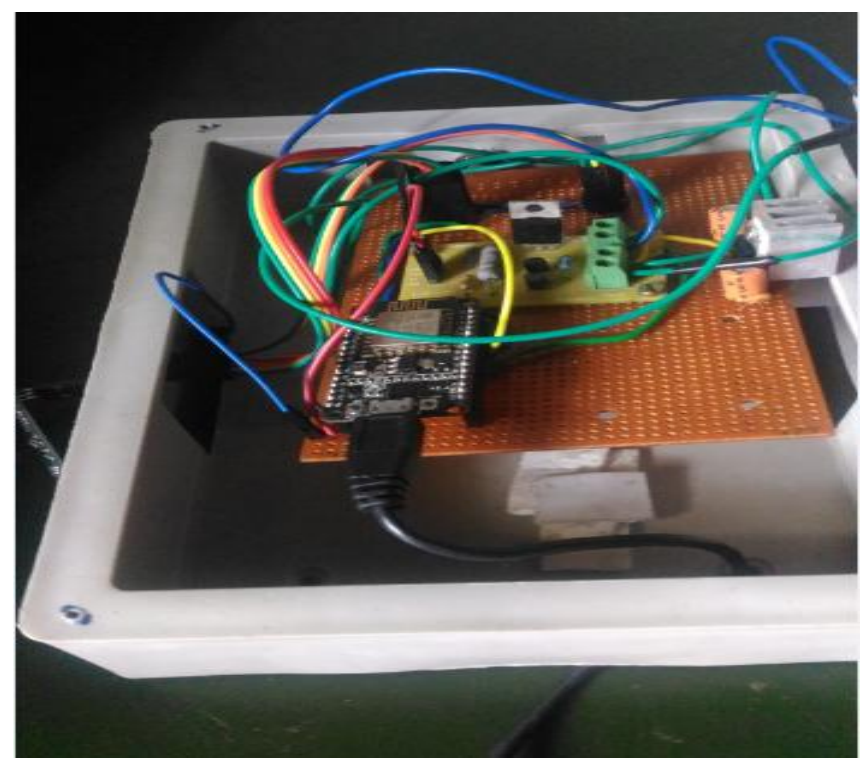

Fig 6. Internal setup in a module

The software part of this work is as important as the hardware part. First of all, the ESP8266 boards need to be loaded with the firmware code. The firmware code is written using Arduino IDE. It is loaded to the ESP8266 boards using the Arduino UNO. The communication between each light module [between each ESP8266 microcontroller] is takes place via MQTT protocol. MQTT stands for Message Queue Telemetry Transport. The MQTT is a machine to machine or IoT connectivity protocol. It is an extremely

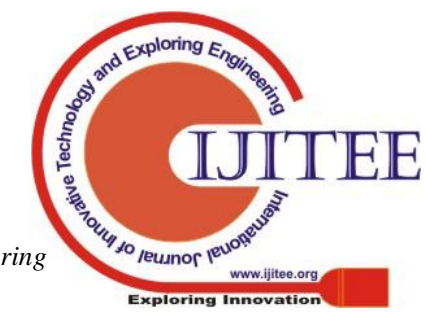


light weight publish/subscribe messaging protocol. The small size, low power usage, minimized data packets and ease of implementation makes MQTT ideal for the IoT world. There are 5 MQTT components namely broker, topic, message, publish and subscribe. Broker is the server that handles the data transmission between the clients. Each message is send via a topic. The topic make the message selective and message is the data that a device receives "when subscribing" from a topic or send "when publishing" to a topic. Publish is the process a device does to send its message to the broker and subscribe is the process where a device does to retrieve a message from the broker. Mosquitto broker is used here for implementing the MQTT protocol.

\section{RESULTS \& DISCUSSIONS}

The MQTT broker application in Google play store creates MQTT broker on our Android phone. It can act as both MQTT broker and MQTT client. The broker can be started and stopped by a single touch on the display. When the broker is in "STOP" condition, the display will be like given in figure 7.

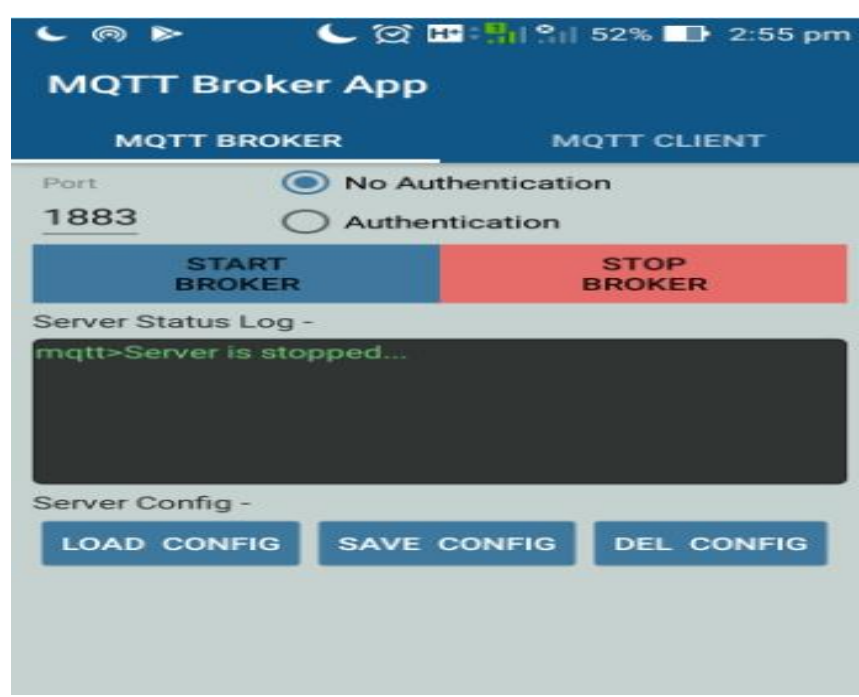

Fig 7. When MQTT broker is stoped

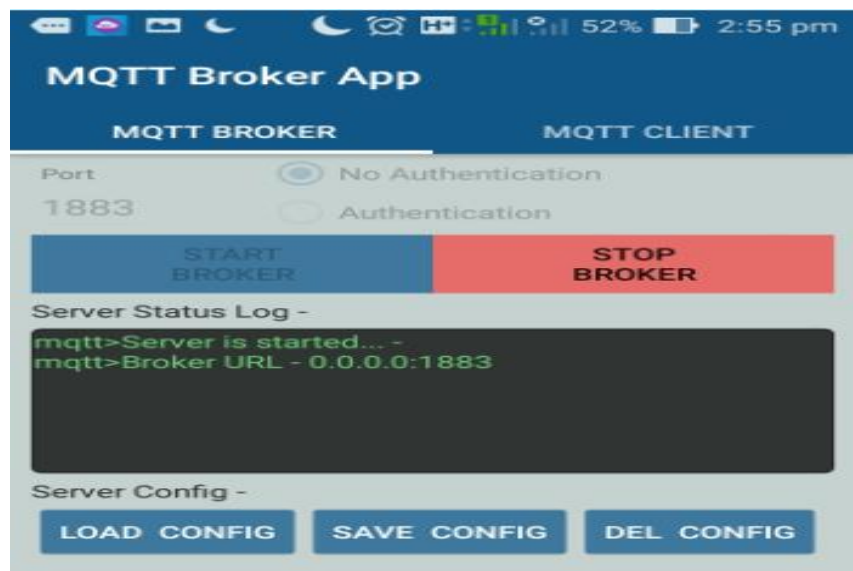

Fig 8. When MQTT broker is under working condition
The MQTT client can be created in the way given in figure 9:

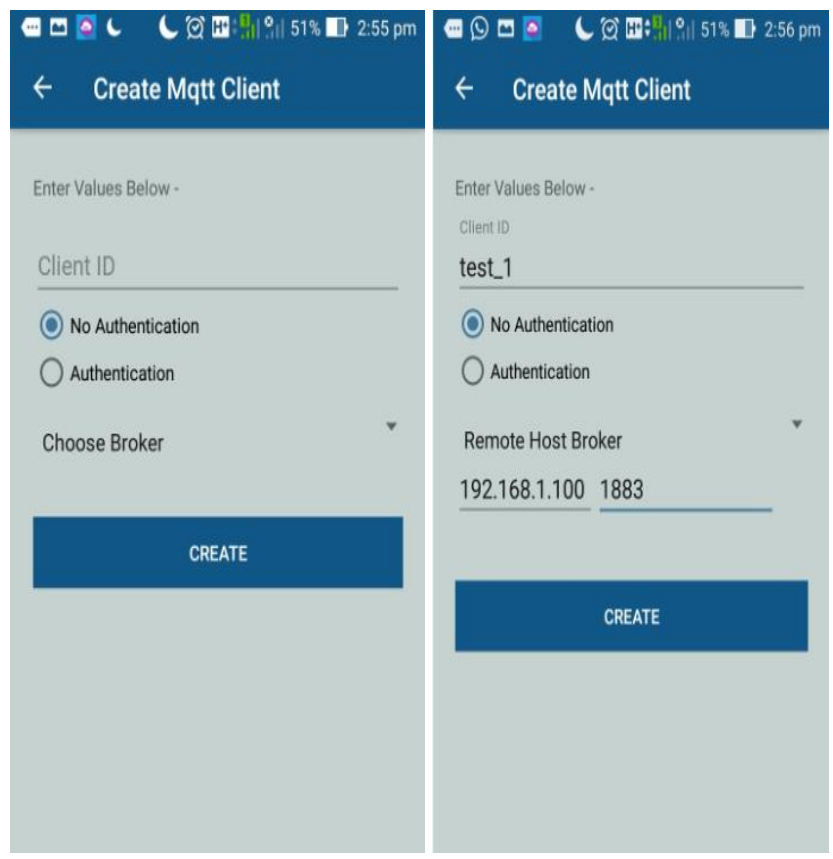

(a)

(b)

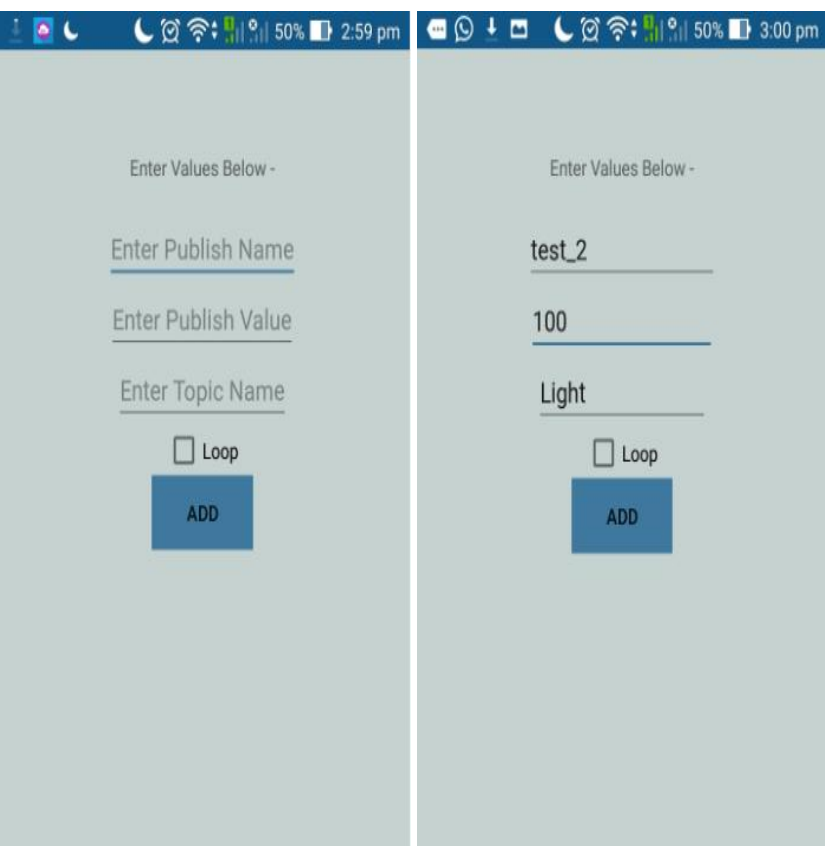

(c)

Fig 9. Creation of MQTT client

An application named MQTT box is available in google chrome. This application connects to a MQTT broker and is able to subscribe and publish to MQTT topics. The below figures describes the MQTT box connection setup in Google chrome. While opening the MQTT box, we get a display like this. Here we have to specify the MQTT client name, protocol, username, password etc. 


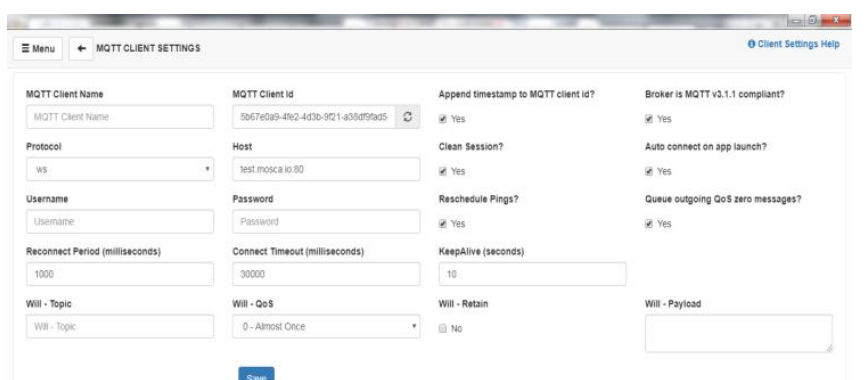

(a)

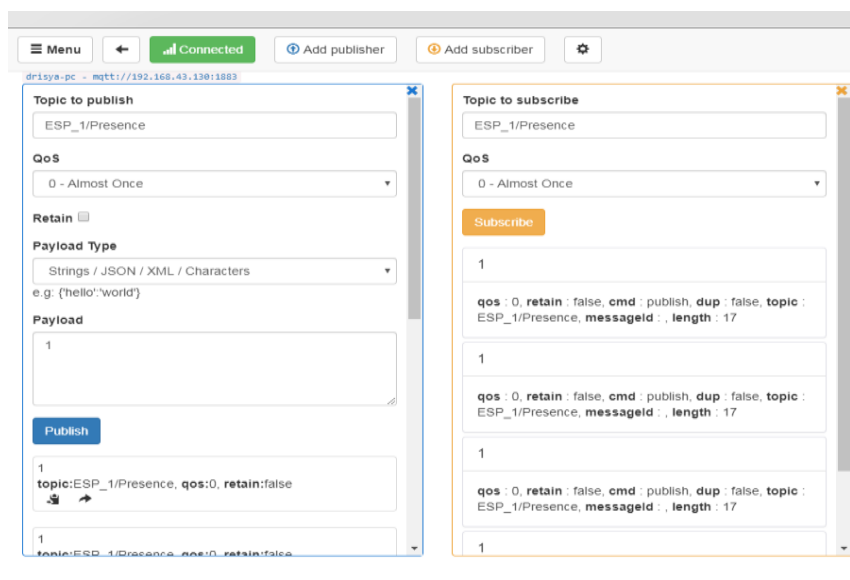

(b)

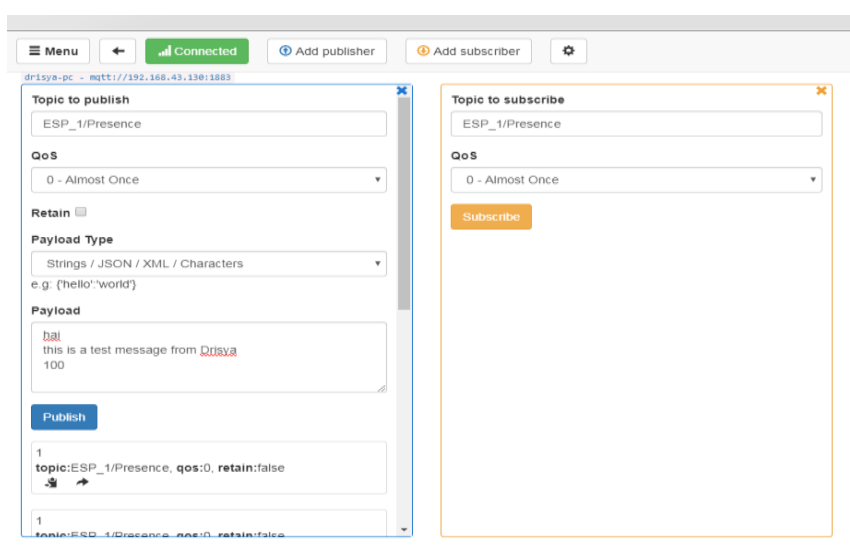

(c)

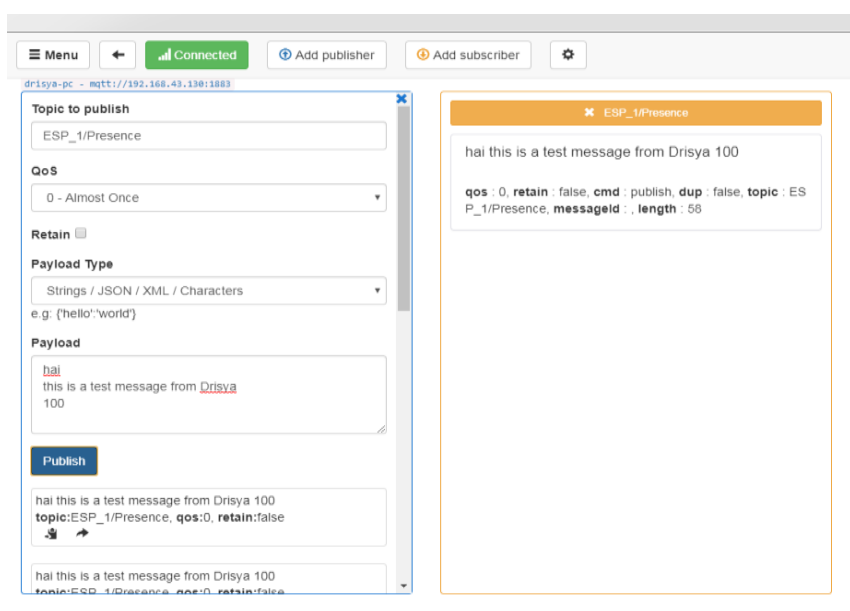

(d)

Fig 10.MQTT box connection setup (a,b,c,d)
Step 1: Start

Step 2: Declare variables Presence, sensor_state, Light, ESP_1/Presence, ESP_2/Presence, ESP_3/Presence. Step 3: State WiFissid, password, MQTT broker ip, MQTT broker port, MQTT username, MQTT password and client name.

Step 4: Publish sensed values from the light sensor and presence detectors.

Step 5: Check "Light" value

$$
\text { ifESP_i/Presence }=1
$$

input the value in luminous to light source

if Light $=\mathrm{j}$, luminous $=1024-\mathrm{j}$

Step 6: If ESP_i/Presence $=0$ and $\left(E S P \_(i-1) /\right.$ Presence $=1$ or ESP_(I+1)/Presence $=1)$

Step 7: stop.

input the value ' 100 ' to light source

\section{CONCLUSIONS}

Green buildings and IoTs are two important concepts in now a days. An IoT based smart home concept have much importance in this era where energy conception and wastage rates are increasing drastically. So an intelligent ambient controller that controls indoor lighting inaccordance with external lighting and human presence requires much importance. IoT communication via MQTT protocol is utilized in this smart home concept. MQTT is an ISO standard publish - subscribe based messaging protocol and requires a message broker.Mosquitto is an Open source message broker that implements the MQTT protocol. Eclipse Mosquitto provides a lightweight server implementation of the MQTT protocol that is suitable for all situations from full power machines to embedded and low power machines. Sensors and actuators, which are often the sources and destinations of MQTT messages, can be very small and lacking in power.

In this work, an efficient intelligent ambient controller for $\underline{\text { LVDC }}$ enabled green building is designed and implemented. Such a system is important due to the increasing usage of energy resources and the energy wastage. The implemented system have the capacity to adjust the light intensity in accordance with the external light and human presence. So that the light modules will light brightly when it is night and the light intensity will be less, when the time is day and there is no human presence.

\section{REFERENCES}

1. Corno, Fulvio and De Russis, Luigi and Bonino, Dario, "Educating internet of things professionals: The ambient intelligence course", IT Professional, IEEE, vol.18, no.6, pp. $50-57,2016$.

2. Leong, Peter and Lu, Liming, "Multiagent web for the internet of things", 2014 International Conference on Information Science \& Applications (ICISA), IEEE, pp.1-4, 2014.

3. Amisha Ashok Karia, Lavina Vijay Budhwani, Vishal SahebraoBadgujar,"IoT key towards automation", International Journal of Computer Applications (0975 8887), vol.113, no. 1, March 2015.

Published By: Blue Eyes Intelligence Engineering 
4. Chen, Hao and Jia, Xueqin and $\mathrm{Li}$, Heng, "A brief introduction to IoTgateway",IET International Conference on Communication Technology and Application (ICCTA 2011)\},IET, pp. 610-613,2011.

5. Kraijak, Surapon and Tuwanut, Panwit," A survey on IoT architectures, protocols, applications, security, privacy, realworld implementation and future trends", IET, 2015.

6. VagishAdhav, S. R. Gulhane, "An IoT Based Monitoring and Control System For Environmental Conditions and Safety In Home”, 2016 IJEDR, vol. 4, issue 4, ISSN: 2321-9939, 2016.

7. Chinju Paul, Amal Ganesh, Sunitha C, "An Overview of IoT Based Smart Homes", Proceedings of the Second International Conference on Inventive Systems and Control (ICISC 2018), IEEE, ISBN:978-1-5386-0806-7, 2018.

8. Zouai, Meftah and Kazar, Okba and Haba, Belgacem and Saouli, Hamza, "Smart house simulation based multi-agent system and internet of things", 2017 International Conference on Mathematics and Information Technology (ICMIT), IEEE, pp. 201-203, 2017.

9. Zouai, M and Kazar, O and Haba, B and Saouli, H and Benfenati, H, "IoT approach using multi-agent system for ambient intelligence", vol.11, no.9, pp.15-32, 2017.

10. Das, SajalKanta and Mukherjee, A, "IoT based smart home management to enhance the services to the occupancies and minimized energy demand by controlling appliances using wireless motes", 2017 International conference on Microelectronic Devices, Circuits and Systems (ICMDCS), IEEE, pp. 1-6, 2017.

11. Wirawan, I Made and Wahyono, IrawanDwi and Idfi, Gilang and Kusumo, GradiyantoRadityo, "IoT Communication System Using Publish-Subscribe", International Seminar on Application for Technology of Information and Communication,pp.61-65,2018.

12. Marques, Goncalo and Pitarma, Rui," An indoor monitoring system for ambient assisted living based on internet of things architecture", International journal of environmental research and public health, vol.13,no. 11,pp.1152,2016.

13. Li, Yanyan and Niu, Pingjuan and Su, Zhengxiao," Design of greenhouse monitoring and control system based on LED lighting",12th China International Forum on Solid State Lighting (SSLCHINA),pp.123-126,2015.

14. Hantrakul, Kittikorn and Pramokchon, Part and Khoenkaw, Paween and Tantitharanukul, Nasi and Osathanunkul, Kitisak," Automatic Faucet with Changeable Flow based on MQTT protocol",2016 International Computer Science and Engineering Conference (ICSEC),pp.1-5,2016.

15. Nuratch, Santi,"Applying the MQTT Protocol on Embedded System for Smart Sensors/Actuators and IoT Applications",15th International Conference on Electrical Engineering/Electronics, Computer, Telecommunications and Information Technology (ECTI-CON),pp.628-631,2018.

16. Chen, Hsiang Wen and Lin, Fuchun Joseph," Converging MQTT resources in ETSI standards based M2M platform",IEEE International Conference on Internet of Things (iThings), and IEEE Green Computing and Communications (GreenCom) and IEEE Cyber, Physical and Social Computing (CPSCom),pp.292-295,2014.

17. Yassein, MuneerBani and Shatnawi, Mohammed Q and Aljwarneh, Shadi and Al-Hatmi, Razan,'Internet of Things: Survey and open issues of MQTT protocol",2017 International Conference on Engineering \& MIS (ICEMIS),pp.1-6, 2017.

18. Atmoko, RachmadAndri and Yang, Daoguo," Online Monitoring \& Controlling Industrial Arm Robot Using MQTT Protocol",IEEE International Conference on Robotics, Biomimetics, and Intelligent Computational Systems (Robionetics),pp.12-16,2018. 